\title{
LANDSCHAFTSPFLEGE IM BLICKFELD ANGEWANDTER GEOGRAPHIE
}

\author{
Karl Ruppert ${ }^{*}$ \\ UREJANJE KRAJINE VLUČI APLIKATIVNE GEOGRAFIJE
}

Izvleček

$U D K$ 911.9:71=30

Prispevek obravnava pokrajino z vidika socialnega razvoja in potenciala za rekreacijo. Podrobno so predstavljene organizacijske oblike urejanja pokrajine na Bavarskem.

Ključne besede: Socialna geografija, aplikativna geografija, prostočasne dejavnosti,; urejanje krajine, regionalni razvoj, Bavarska.

\section{LANDSCAPE ORDERING IN THE LIGHT OF APPLIED GEOGRAPHY}

\section{Abstract}

Landscape is discussed, viewed from the standpoint of social development, and as a potential for recreation. Presented in detail are the organizational forms of landscape ordering in Bavaria.

Key words: Social geography; Applied geography; Leisure activities; Landscape ordering; Regional development; Bavaria.

\footnotetext{
"Dr., prof., Institut für Wirtschaftsgeographie, Ludwig Maximillian Universität. Ludvigstrasse 28 VG, D-80539 München, Deutschland
} 


\section{DER RAUM IM SPIEGEL GESELLSCHAFTLICHER ENTWICKLUNG}

Es gehört zum Grundbestand sozialgeographischer Erkenntnisse, daß Raumstruk turen und räumliche Prozeßabläufe in starkem Maße von der Entwicklung der Gesellschaft abhngig sind. Schon vor fast 30 Jahren hatte Vl. Klemenčič in der Hartke-Festschrift (1) auf den Zusammenhang zwischen dem Wandel der Arbeiter-Bauern-Betriebe und dem Zerfall der Agrarstruktur bis hin zur Entwicklung der Sozialbrache verwiesen. Das slowenische Beispiel zeigte gewissermaßen in einem Zeitraffer den Wandel von der Agrar- zur Industrie- und Dienstleistungsgesellschaft, wie er in seiner räumlichen Komponente über Jahrzehnte im mitteleuropäischen Raum beobachtet werden konnte. Dieser Wandel fand seinen sichtbaren Niederschlag u.a. in der Veränderung der Bodennutzung, zum Teil in der Aufgabe jeglicher Nutzung.

Im Zuge dieser Entwicklung traten neue Grundperspektiven auf, die für die Gestaltung der Raumstrukturen immer wichtiger wurden:

- das Freizeitverhalten trat als neue Grundfunktion gleichwertig neben Arbeiten, Wohnen, Versorgen usw. Neue Formen der Flächennutzung sowie der Wohnstandortspaltung (Freizeitwohnsitze) wurden für ländliche Räume bedeutsam.

- Das wachsende Umweltbewußtsein, der Schutzgedanke, führten zu steigender Wertschätzung der natürlichen Potentiale und der Freiräume.

Die Anwendung neuer Technologien, der Düngemitteleinsatz und Maßnahmen zur Schädlingsbekämpfung führten dazu, daß bei stark steigenden Erträgen ein immer geringerer Teil der Erwerbstätigen ausreichte, um immer mehr Nahrungsmittel zumeist noch auf kleineren Flächen zu produzieren. Die Auswirkungen für die Agrarwirtschaft lagen auf der Hand: in der EG z.B. sank die Zahl der landwirtschaftlichen Erwerbsbevölkerung von 1960 - 1990 von $21 \%$ auf $6,6 \%$, wobei dieser Durchschnitt zwischen den Extremwerten 2,2\% im Vereinigten Königreich bzw. 3,4\% in der Bundesrepublik Deutschland und $25,3 \%$ in Griechenland schwankte. Dieser Schrumpfungsprozeß spiegelt sich auch im weiter rückläufigen Anteil der landwirtschaftlichen Produktion am Bruttoinlandsprodukt, der in der EG 1990 nur noch etwa $3 \%$ betrug.

Von diesen säkularen Änderungen blieb die Wertschätzung des primären Sektors nicht unbeeinflußt. Sehr bald setzte sich auch außerhalb der Agrarwirtschaft die Erkenntnis durch, daß ihre Bedeutung nicht an diesen Quoten allein gemessen werden darf. Wurde zunächst nur auf die von der Landwirtschaft bewirtschafteten Flächen verwiesen, so fiel die Aufmerksamkeit bald auf Leistungen der Landwirtschaft, die nicht unmittelbar der Nahrungsmittel- und Rohstoffproduktion zuzurechnen waren. Begriffe wie "außerökonomische Leistungen", "Wohlfahrtsfunktionen", "nicht marktgängige Leistungen" und dergleichen mehr verwiesen auf eine Vielzahl von positiven Begleiterscheinungen, die mit der landwirtschaftlichen Produktion ver- 
bunden sind. Mehr und mehr wurden die Leistungen der Agrarwirtschaft zur Erhaltung und Gestaltung der Kulturlandschaft anerkannt. Ihre Vielschichtigkeit reicht von wirtschaftlichen, sozialkulturellen bis zu ökologischen Auswirkungen, von der Pflege erholungsorientierter Landschaften bis zur Erhaltung naturnaher Raumelemente und traditioneller Landschaftsbilder (2).

Von der sinkenden Zahl landwirtschaftlicher Betriebe und der damit häufig verbundenen Aufgabe der Flächennutzung wurde auch das Erscheinungsbild der Kulturlandschaft beeinflußt. Dies betrifft besonders Veränderungen der Vegetation, da unter geoökologischen Gesichtspunkten Mitteleuropa in der Zone eines natürlichen Waldkleides liegt. Das Zuwachsen nicht mehr oder unterbestoßener Almflächen ist im Hochgebirge der beste Beleg für diese Entwicklung. Erinnert sei aber auch an Veränderungen in den Mittelgebirgsbereichen, z.B. im Spessart, wo schon um die Mitte dieses Jahrhunderts die Aufgabe der mit der Wiesenbewässerung verbundenen Grünlandnutzung und ihre Konsequenzen diskutiert werden mußten. Der Ablauf von Sukzessionsstadien war mehrfach Gegenstand vegetationsgeographischer Forschungen. Die Diskussion um die "Verfinsterung" der Landschaft begann. Hohe Aufforstungsprämien der EG trugen ihren Teil dazu bei.

\section{LANDSCHAFT ALS FREIZEITPOTENTIAL}

Fragt man nach der Einschätzung solcher Entwicklungen, dann wird die Antwort je nach Bewertungsgesichtspunkt sehr heterogen ausfallen. Wählt man hier den Blickpunkt des Tourismus oder den Wunsch der Erhaltung des gegenwärtigen Landschaftsbildes als Freizeitpotential, dann sollte im Hinblick auf eine naturbetonte Freiraumerholung, die in den vergangenen Jahrzehnten zunehmend an Bedeutung gewonnen hat und die mit zur Erhaltung zahlreicher bäuerlicher Existenzen beitrug, eine kontinuierliche Landschaftspflege durchgeführt werden. Gerade die Ergebnisse der Tourismusforschung haben gezeigt, daß der Aufenthalt in der freien Natur zu einem wichtigen Reisemotiv geworden ist. Freizeitverhalten ist heute als eine echte Flächennutzung zu betrachten (3).

Die Attraktivität von Landschaften für das Freizeitverhalten ist von vielen Einzelkomponenten abhängig, z.B. vom sozialen Umfeld, infrastruktureller und naturbedingter Ausstattung, Umweltsituation, Gästebetreuung, Erreichbarkeit usw. Je nach der auszuübenden Freizeitaktivität sind die Anforderungen an den Raum unterschiedlich. In aller Regel sind Merkmale wie Vielfalt und Strukturiertheit von Räumen positiv zu bewerten. Brachflächen werden keineswegs generell als unästhetisch empfunden, Nebeneffekte aber wie Verfall von Wegen, von baulichen Einrichtungen, Zuwachsen von Almflächen usw. werden oft als eine gewisse 
Verwahrlosung der Landschaft angesehen und damit als Beeinträchtigung der Erholungseignung. Auf die Bedeutung der Flächennutzung für die Erhaltung des durch die Grünlandnutzung im Hochgebirge geschaffenen neuen ökologischen Gleichgewichts sei hier nur am Rande hingewiesen.

Forschungen zum Erlebniswert der Landschaft werden in der Literatur mehrfach bezüglich des Waldanteiles diskutiert. Schon in den 60er-Jahren zeigten unsere Naherholungsuntersuchungen im Osten von München, daß große geschlossene Waldflächen weniger attraktiv für den Erholungssuchenden sind, während der Waldrand oder größere Lichtungen im Wald sehr häufig von den Naherholern aufgesucht wurden.

Bezüglich eines optimalen Bewaldungsgrades unter Erholungsgesichtspunkten fassen Ammer/Pröbstl (4) die Ergebnisse mehrerer Forschungsarbeiten dahingehend zusammen, daß sie den "optimalen" Waldanteil mit 50 - 70\% angeben. Solche Verallgemeinerungen sind jedoch aus mehrfachen Gründen problematisch, muß doch die Verteilung des Waldes über die Fläche bei gleichem Anteilsquotienten durchaus nicht identisch sein. Größere geschlossene Waldflächen, die für Naherholer weniger interessant sind, können andererseits für eine Kurerholung positiver bewertet werden. Schließlich darf man auch den geländeklimatischen Einfluß größerer Waldflächen nicht übersehen.

In Anbetracht der Bedeutung des Landschaftsbildes im Tourismus und im Hinblick auf weiterhin rückläufige Zahlen landwirtschaftlicher Betriebe gewinnt die Offenhaltung von Freiräumen und damit die Landschaftspflege gerade in Tourismusgebieten an Bedeutung. Bereits 1991 gab es in Bayern 4 Gemeinden ohne einen einzigen landwirtschaftlichen Betrieb und 20 Gemeinden ohne einen Haupterwerbsbetrieb. Bei der Fortsetzung dieser Entwicklung kann die Fortführung der Flächennutzung durchaus zu einem Problem werden. Inzwischen hat sich die Anerkennung der Landschaftspflegeleistungen durch die Landwirtschaft weitgehend durchgesetzt. Ihre Honorierung ist im In- und Ausland ein vielfach erprobtes agrarpolitisches Instrument, zumal sie häufig von Rationalisierungs- und Gewinnverzicht begleitet sind (5). Die Landespflege d. h. Maßnahmen zur Pflege und Erhaltung ökologischer und landschaftlicher Vielfalt, auch Renaturierung naturnaher Lebensräume hat auch im gesellschaftspolitischen Blickfeld eine feste Position bezogen. Sehr plastisch dokumentiert sich dieser Sachverhalt in der Landbereitstellung und den Investitionen für Naturschutz und Landschaftspflege bei den Verfahren der ländlichen Neuordnung in Bayern (Flurbereinigung) (vgl. Abb. 1). 
Investitionen für Naturschultz und Landschaftspflege

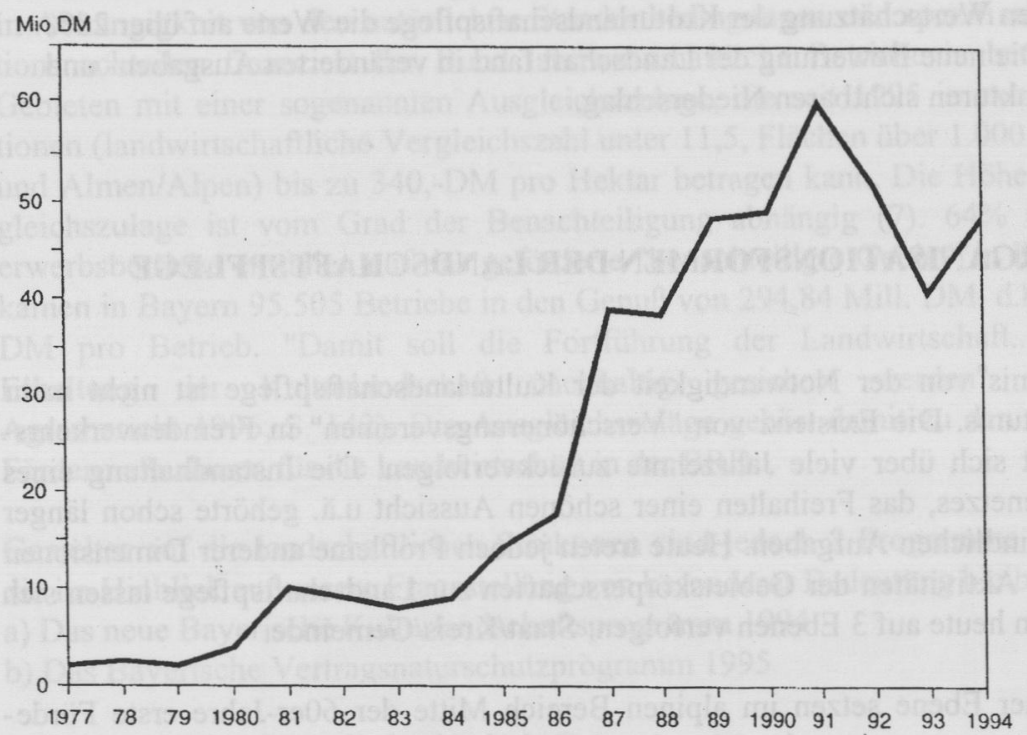

Landerbereitstellung für Naturschutz und Landschaftspflege

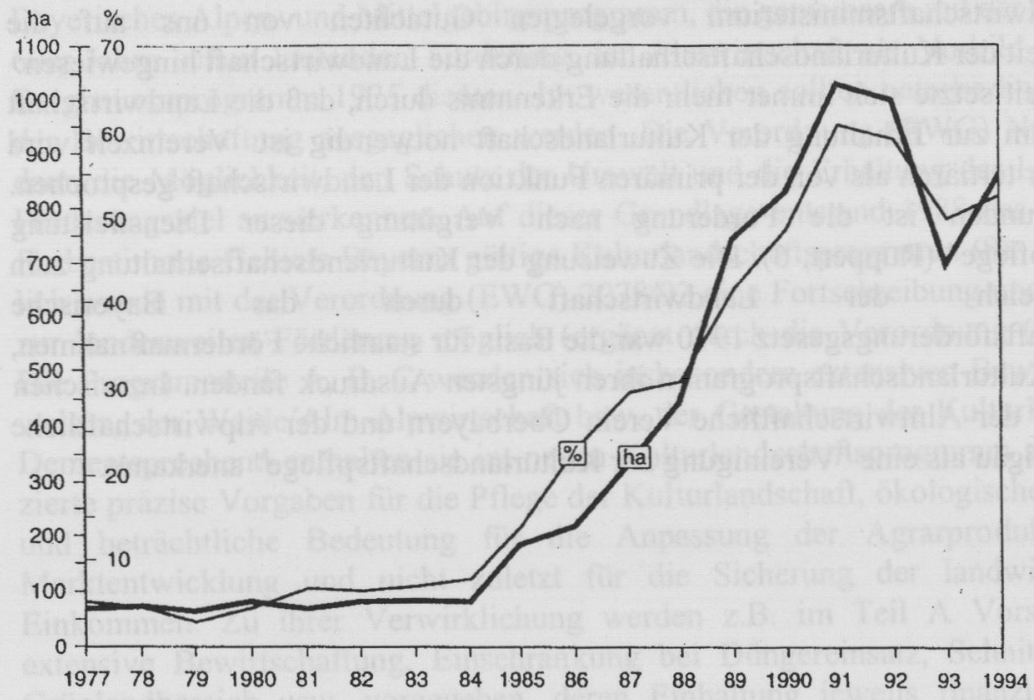

nach StMELF, Referat N 5, 5/91 bzw. E 5, 2/96 
Waren es noch bis in die frühen 80 er Jahre nur 3 - 5\% der Investitionen und weniger als $10 \%$ der Flächen, die für die o.a. Maßnahmen eingesetzt wurden, so wuchsen mit der steigenden Wertschätzung der Kulturlandschaftspflege die Werte auf über 20\% bzw. 50\%. Die neue Bewertung der Landschaft fand in veränderten Ausgaben- und Nutzungsstrukturen sichtbaren Niederschlag.

\section{ORGANISATIONSFORMEN DER LANDSCHAFTSPFLEGE}

Die Erkenntnis von der Notwendigkeit der Kulturlandschaftspflege ist nicht mehr neuesten Datums. Die Existenz von "Verschönerungsvereinen" in Fremdenverkehrsgebieten läßt sich über viele Jahrzehnte zurückverfolgen. Die Instandhaltung eines Wanderwegenetzes, das Freihalten einer schönen Aussicht u.ä. gehörte schon länger zu den gemeindlichen Aufgaben. Heute treten jedoch Probleme anderer Dimensionen auf. Gezielte Aktivitäten der Gebietskörperschaften zur Landschaftspflege lassen sich u.a. in Bayern heute auf 3 Ebenen verfolgen: Staat/Kreis/Gemeinde.

Auf staatlicher Ebene setzen im alpinen Bereich Mitte der 60er-Jahre erste Förderungsmaßnahmen zugunsten der Almwirtschaft ein, die schon damals als Maßnahmen zur Kulturlandschaftserhaltung verstanden wurden. Zur Förderung der Jungviehsömmerung wurden 1966 bereits 263.000,-DM ausgesetzt, ein Wert, der bis zum Jahr 1970 auf über 900.000,-DM anstieg. Damals wurde in einem 1971 dem Bayerischen Landwirtschaftsministerium vorgelegten Gutachten von uns auf die Notwendigkeit der Kulturlandschaftserhaltung durch die Landwirtschaft hingewiesen: "In letzter Zeit setzte sich immer mehr die Erkenntnis durch, daß die Landwirtschaft im Alpenraum zur Erhaltung der Kulturlandschaft notwendig ist. Vereinzelt wird mehr von der tertiären als von der primären Funktion der Landwirtschaft gesprochen. Damit verbunden ist die Forderung nach Vergütung dieser Dienstleistung Landschaftspflege" (Ruppert, 6). Die Zuweisung der Kulturlandschaftserhaltung zum Aufgabenbereich der Landwirtschaft durch das Bayerische Landwirtschaftsförderungsgesetz 1970 war die Basis für staatliche Fördermaßnahmen, die u.a. im Kulturlandschaftsprogramm ihren jüngsten Ausdruck fanden. Inzwischen wurden auch der Almwirtschaftliche Verein Oberbayern und der Alpwirtschaftliche Verein im Allgäu als eine "Vereinigung zur Kulturlandschaftspflege" anerkannt. 


\section{Spezielle Förderprogramme}

In Abhängigkeit von den natürlichen Standortbedingungen und spezifischen Produktionsnachteilen finanziert der Bund landwirtschaftliche Betriebe in benachteiligten Gebieten mit einer sogenannten Ausgleichszulage, die seit 1995 in extremen Situationen (landwirtschaftliche Vergleichszahl unter 11,5, Flächen über 1.000 Meter Höhe und Almen/Alpen) bis zu 340,-DM pro Hektar betragen kann. Die Höhe dieser Ausgleichszulage ist vom Grad der Benachteiligung abhängig (7). $64 \%$ aller Haupterwerbsbetriebe entfallen auf das geförderte "benachteiligte Gebiet" in Bayern. 1995 kamen in Bayern 95.505 Betriebe in den Genuß von 294,84 Mill. DM, d.h. ca. 3.000,DM pro Betrieb. "Damit soll die Fortführung der Landwirtschaft... sowie die Erhaltung der Kulturlandschaft nachhaltig gesichert werden" (Bayerischer Agrarbericht 1996, S. 142). Die Ausgleichszulage gehört damit zu den wesentlichen Fördermaßnahmen für die Landwirtschaft in der BRD.

Gezielter auf die landschaftlichen Strukturen sind jedoch 2 Programme ausge richtet, die im Hinblick auf unsere Fragestellung von besonderer Bedeutung bleiben:

a) Das neue Bayerische Kulturlandschaftsprogramm 1994

b) Das Bayerische Vertragsnaturschutzprogramm 1995

\section{a) Das neue Bayerische Kulturlandschaftsprogramm}

Die im Gesetz zur Förderung der bayerischen Landwirtschaft 1970 enthaltene Zielsetzung der Erhaltung des ländlichen Raumes als Kulturlandschaft war die Basis für eine Reihe von Förderprogrammen wie z.B. Bayerisches Grünlandprogramm (1972), Bayerisches Alpen- und Mittelgebirgsprogramm, die gemeinsam mit der seit Mitte der 60er-Jahre schon laufenden Förderung der Almwirtschaft als Vorbild für das EGBergbauernprogramm 1975 dienten. Im wesentlichen sollten naturbedingte Nachteile der Bewirtschaftung ausgeglichen werden. Die Verordnung (EWG) Nr. 797/85 bot dann die Möglichkeit, den Schutz der Umwelt und die Erhaltung der Landschaft als Förderungsziel anzuerkennen. Auf dieser Grundlage entstand 1988 das zunächst nur für bestimmte Gebiete Bayerns gültige Kulturlandschaftsprogramm (8). 1992 schließlich wurde mit der Verordnung (EWG) 2078/92 eine Fortschreibung und Ausweitung zur landesweiten Förderung möglich (ergänzt durch die Verordnung (EG) 746/96). Die Programmteile A, B, C wenden sich insbesondere extensiven Bewirtschaftungsweisen, der Weide/Alm-Alpwirtschaft bzw. der Gestaltung der Kulturlandschaft zu. Dementsprechend enthalten sie im neuen Kulturlandschaftsprogramm stark differenzierte präzise Vorgaben für die Pflege der Kulturlandschaft, ökologische Maßnahmen und beträchtliche Bedeutung für die Anpassung der Agrarproduktion an die Marktentwicklung und nicht zuletzt für die Sicherung der landwirtschaftlichen Einkommen. Zu ihrer Verwirklichung werden z.B. im Teil A Vorschriften über extensive Bewirtschaftung, Einschränkung bei Düngereinsatz, Schnittzeitpunkt im Grünlandbereich usw. vorgegeben, deren Einhaltung jeweils finanziell durch ein Leistungsentgelt honoriert werden. Im Teil B steht die Förderung der Infrastruktur auf 
Flächen der Alm- und Weidewirtschaft bis zur Teilfinanzierung der Hubschrauberversorgung bei fehlender Wegeerschließung an, während im Teil C z.B. Schutzpflanzungen, Feldgehölze und überbetriebliche Maßnahmen zur Gestaltung der Kulturlandschaft gefördert werden. Genauere Einzelkriterien bezüglich Laufzeit, Honorierung, Maßnahmen vergleiche beiliegende Tabelle 1a, 1b, 1c.

Tabelle la

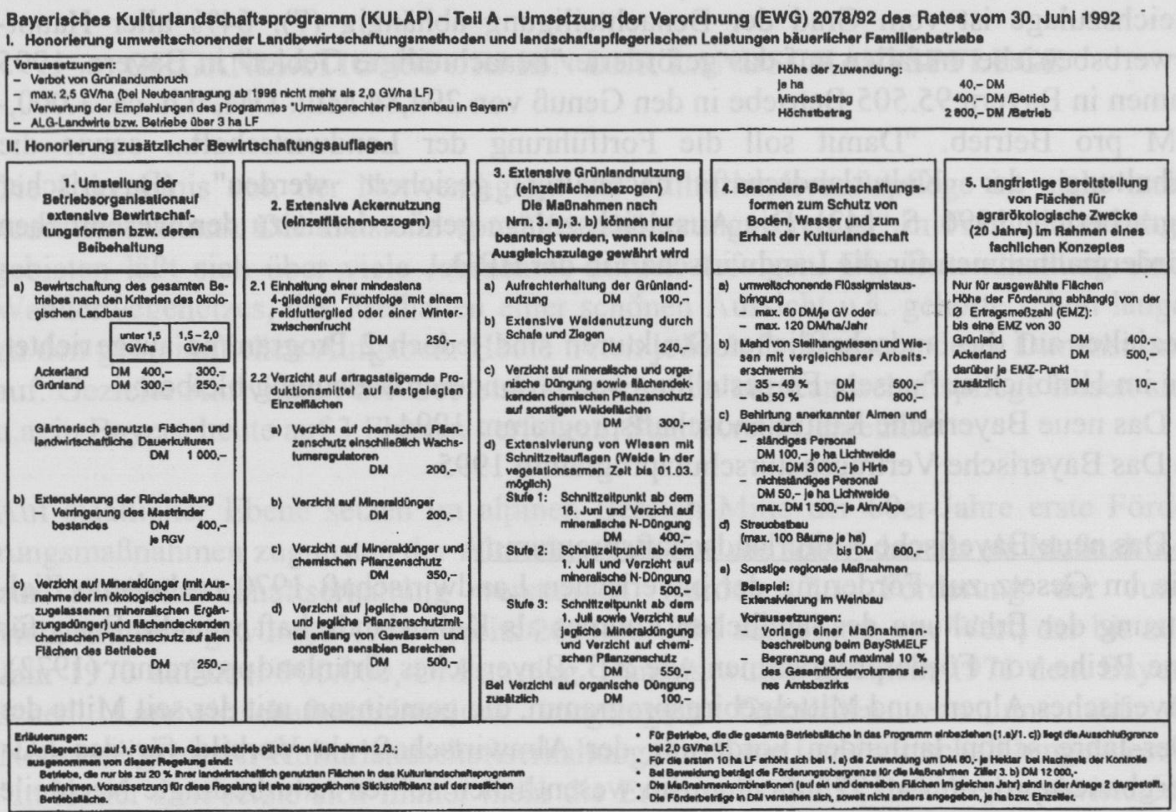

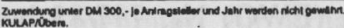

Stant oupe 
Tabelle $1 b$

BAYERISCHES KULTURLANDSCHAFTSPROGRAMM

sulty vern 18 OS.1950 - nach den Richtlinien des Bayerischen Staatsministeriums für Ernăhrung, Landwirtschaft und Forsten -

Teil 8: Weide-/Alm-/Alpwirtschaft

\begin{tabular}{|c|c|c|c|c|c|c|}
\hline $\begin{array}{l}\text { Zweck der Maß- } \\
\text { nahme }\end{array}$ & \multicolumn{6}{|c|}{ 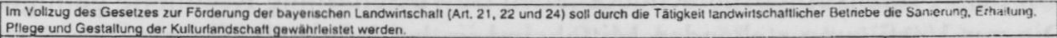 } \\
\hline $\begin{array}{l}\text { Gegenstand der } \\
\text { Förderung }\end{array}$ & \begin{tabular}{l}
\multicolumn{1}{c}{2.1} \\
Neubau, Reparaturen, \\
Verbesserungen von \\
Alm-/Alpgebäuden aut \\
anerkannten Almen \\
Alpen
\end{tabular} & $\begin{array}{l}2.2 \\
\text { Schaffung von Weide- } \\
\text { einrlehtungen (z. B. Vieh- } \\
\text { schutzhütten, Weldezdu- } \\
\text { ne, Welderoste, Wasser- } \\
\text { versorgung); aut aner- } \\
\text { kannten AlmenvAlpen } \\
\text { auch die Wiederherstel- } \\
\text { lung }\end{array}$ & $\begin{array}{l}2.3 \\
\text { Bau von AnschluBwegen } \\
\text { im Bereleh anerkannter } \\
\text { Almen/Alpen }\end{array}$ & \begin{tabular}{l}
\multicolumn{1}{c}{2.4} \\
Beschaffung von fabrik- \\
neuen Motormähern, \\
Motorheu- und Anbau. \\
heumaschinen
\end{tabular} & \begin{tabular}{l}
\multicolumn{1}{c}{2.5} \\
Zaununterhalt aut ver- \\
blelbender Lichtweide- \\
flikche nach Trennung \\
von Wald und Weide
\end{tabular} & $\begin{array}{l}\text { 2.6 } \\
\text { Grundversorgung von } \\
\text { anerkannten Almen' } \\
\text { Alpen mit Hubschrouber }\end{array}$ \\
\hline $\begin{array}{l}\text { Zuwendungs- } \\
\text { emptänger }\end{array}$ & \multicolumn{6}{|c|}{$\begin{array}{l}\text { - Landwirtschaftliche Unternehmer im Sinne des } \$ 1 \text { des Gesetzes úber eine Altershilte für Landwirte (GAL). } \\
\text { - Inhaber von landwirtschattlichen Betrieben unter dieser Grenze, wenn sie eine Holstelle mil Betriebsgebäuden besitzen und Landwinschait eindeutig zu Enwerbszwek. } \\
\text { ken betreiben. } \\
\text { - Kooperationen (z. B. Alm-/Alp.Weidegenossenschatten) im Namen und Aultrag ihrer antragsberechtigten Mitglieder. }\end{array}$} \\
\hline \multirow[t]{2}{*}{$\begin{array}{l}\text { Forderungs- } \\
\text { voraussetzungen }\end{array}$} & \multicolumn{6}{|c|}{ 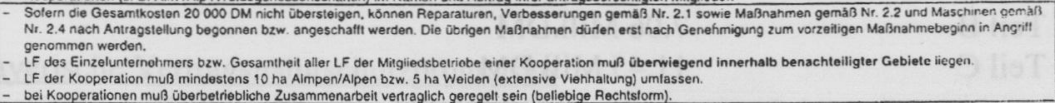 } \\
\hline & $\begin{array}{l}\text { in Einzelunternehmen } \\
\text { Neubau nur autgrund ei- } \\
\text { nes Elementarschadens } \\
\text { oder ais FolgemaßBnahme } \\
\text { einer Trennung von Wald } \\
\text { und Weide }\end{array}$ & 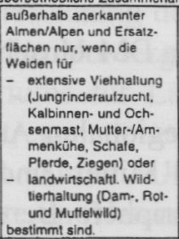 & & $\begin{array}{l}\text { Nutzung von schwer } 24 \\
\text { bewintschallenden Flachen } \\
\text { (Hang:. Feuchtliachen. } \\
\text { Buckelwiesen) }\end{array}$ & $\begin{array}{l}\begin{array}{l}\text { Trennung von Wald und } \\
\text { Weide }\end{array} \\
\text { a }\end{array}$ & $\begin{array}{l}\text { Alm/Aipe wegemäßjig nicht } \\
\text { erschiossen }\end{array}$ \\
\hline \multirow[t]{2}{*}{$\begin{array}{l}\text { Höhe der } \\
\text { Förderung }\end{array}$} & $\begin{array}{l}\text { bis zu } 50 \% \text { der zuwen. } \\
\text { dungsfahigen Kosten. } \\
\text { max. } 90000 \text { DM }\end{array}$ & $\begin{array}{l}\text { bis zu } 50 \% \text { der zuwen. } \\
\text { dungstanigen Kosten. } \\
\text { max. } 20000 \text { DM }\end{array}$ & $\begin{array}{l}\text { bis zu } 50 \% \text { der zuwen. } \\
\text { dungslähigen Kosten. } \\
\text { max. } 20000 \mathrm{OM}\end{array}$ & \multirow{2}{*}{$\begin{array}{l}700 \text { DM je Molormáher } \\
900 \text { DM le Molorheuma. } \\
\text { schine } \\
500 \text { DM je Anbauheu- } \\
\text { maschine }\end{array}$} & \multirow[t]{2}{*}{$\begin{array}{l}800 \text { DM je km Zaun als } \\
\text { einmalige Pauschale }\end{array}$} & \multirow[t]{2}{*}{$\begin{array}{l}\text { bis zu } 50 \% \text { der zuwen- } \\
\text { dungstâhigen Autwendut- } \\
\text { gen }\end{array}$} \\
\hline & $\begin{array}{l}\text { ais Folgema } \\
\text { bis zu } 60 \% \text { der zuwen- } \\
\text { dungstahigen Kosten, } \\
\text { max. } 115000 \text { DM }\end{array}$ & $\begin{array}{l}\text { nahmen zur Trennung von W } \\
\text { bis zu } 100 \% \text { der zuwen- } \\
\text { dungstâhigen Kosten. } \\
\text { max. } 40000 \mathrm{DM}\end{array}$ & $\begin{array}{l}\text { ald und Weide } \\
\text { bis zu } 100 \% \text { der zuwen. } \\
\text { dungstahigen Kosten. } \\
\text { max. } 40.000 \text { DM }\end{array}$ & & & \\
\hline
\end{tabular}

Tabelle 1c

\section{Bayerisches Kulturlandschaftsprogramm - Teil C}

\begin{tabular}{|c|c|c|c|c|c|}
\hline & 2.1 & 2.2 & 2.3 & 2.4 & 2.5 \\
\hline Gegenstand der Forderung & \begin{tabular}{|l|} 
Anlage/Erneuerung von \\
Schutzpflanzungen, \\
Feldgehölzen, Streuobst- \\
bestanden, enschlieBlich \\
Anlage von Waldsaumge- \\
sellschaften aut der land- \\
winschahtichen Betriebsfla- \\
Che. ausgenommen forst. \\
wirtschaftliche Nutzflache \\
\end{tabular} & $\begin{array}{l}\text { Pllege von bestehenden } \\
\text { Schutzpflanzungen und } \\
\text { Feldgehözzen zur Erhal: } \\
\text { tung und Sicherung enes } \\
\text { funktionsgerechien Bestan- } \\
\text { des }\end{array}$ & $\begin{array}{l}\text { MaßBnahmen zur Siche- } \\
\text { rung extensiver landwirt. } \\
\text { schaftlicher Nutzungs. } \\
\text { formen, z. B. Entbu- } \\
\text { schungen, sowie Anlage } \\
\text { sonstiger nurzungsbezo. } \\
\text { gener Landschansbe. } \\
\text { standtelle }\end{array}$ & $\begin{array}{l}\text { Umwandlung von Acker- } \\
\text { land In Grünland ein. } \\
\text { schlieBlich Anlage von } \\
\text { Grünland ais Randstreiten } \\
\text { an Gowassern, forstwin. } \\
\text { schathich genutzien Fla. } \\
\text { chen und sonstigen nut. } \\
\text { zungsberogenen Land- } \\
\text { schaftsbestandteilan } \\
\end{array}$ & $\begin{array}{l}\text { Überbetriebliche Maß- } \\
\text { nahmen zur Erhaltung. } \\
\text { Pflege und Gestaltung } \\
\text { der Kulturlandschah }\end{array}$ \\
\hline $\begin{array}{l}\text { Förderungs. } \\
\text { voraussetzungen }\end{array}$ & 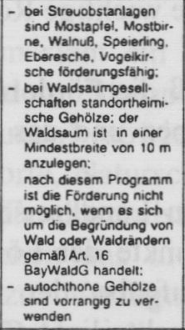 & $\begin{array}{l}\text { Pliegekonzept der Ablei- } \\
\text { lung B I des Amtes fir } \\
\text { Landwirtschath und Ernah- } \\
\text { rung }\end{array}$ & $\begin{array}{l}\text { keine Maschinen. Viet. } \\
\text { odder Gebaudeförderung: } \\
\text { lediglich 2. B. Auszaunung }\end{array}$ & $\begin{array}{l}\text { - mindestens } 10 \text { Jahre } \\
\text { Grülandnutzung } \\
\text { - kein Grunniandumbruch } \\
\text { aut den Ubrigen Be- } \\
\text { triebstlachen }\end{array}$ & $\begin{array}{l}\text { - nach LwFöG anerkannte } \\
\text { privatrechtliche Zusam. } \\
\text { menschlusse von Inna. } \\
\text { bern landwirtschattlicher } \\
\text { Betriabe } \\
\text { - Fünfliahres- und Jahres. } \\
\text { plan }\end{array}$ \\
\hline Hohe der Förderung & 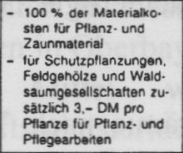 & $\begin{array}{l}\text { pauschal } 40,-D M \text { pro at } \\
\text { und Ptlegagang }\end{array}$ & $\begin{array}{l}70 \% \text { der zuwendungstâhi. } \\
\text { gen Kosten }\end{array}$ & $\begin{array}{l}\text { pauschal } 2 \text { 000.- DM pro } \\
\text { Hektar } \\
\text { als Randstreifen: } \\
\text { pauschal } 2 \text { 500.- DM pro } \\
\text { Hektar }\end{array}$ & $\begin{array}{l}\text { bis zu } 70 \% \text { der zuwen. } \\
\text { dungstahigen Kosten }\end{array}$ \\
\hline
\end{tabular}

Bemerkungen: Antragstellung beim Amt für Landwirtschatt und Ernahrung

Stant $09 / 93$ 
Von großer Bedeutung war auch die erstmalige Einführung eines gene rellen Bewirtschaftungsentgeltes von zunächst 40,-DM pro Hektar (ab1997: 60,-DM pro Hektar) bei Einhaltung spezieller Kriterien z.B. Begrenzung des Viehbesatzes usw. Dadurch soll dem Prinzip einer flächendeckenden Bewirtschaftung Rechnung getragen werden. Die zur Verfügung stehenden Mittel sind in den letzten Jahren beträcht lich gesteigert worden, wobei 50\% von der EU getragen werden. Insgesamt betrugen 1996 die Fördermittel für das

- Bewirtschaftungsentgelt 108.63 Mill.DM

- Teil A

124.28 Mill.DM

- Teil B

7.06 Mill.DM

- Teil C

Für die Zeitspanne 1993 - 1997 sollen ca. 1.2 Mia.DM einschließlich der 50\% EUMittel bereit stehen (9).

Die Akzeptanz dieses Programmes besitzt unter regionalen Aspekten eine deut liche Differenzierung. Generell läßt sich festhalten, daß Mittel- und Hochgebirgsbereiche (einschließlich Vorland) also im wesentlichen periphere Bereiche Bayerns an den flächenbezogenen Maßnahmen überdurchschnittlich beteiligt sind, ergänzt um einige Ackerbaugebiete meist geringerer Produktionsfähigkeit. Der Anteil der 1995 geförderten Fläche liegt hier zum Teil beträchtlich über dem bayerischen Durchschnitt von $10,5 \%$ (1995) LF. Besonders intensive Beteiligung an einzelnen Teilprogrammen fallen auf:

- Ökologischer Landbau und extensive Bewirtschaftung des Gesamtbetriebes: Bayerischer Wald sowie Alpen und Alpenvorland

- Extensive Grünlandnutzung: Periphere Mittelgebirge sowie Alpen und Teile des Alpenvorlandes:

- Extensive Ackernutzung: Unter- und Oberfranken sowie Teile der Oberpfalz.

Neben unterschiedlicher natürlicher Ausstattung spielen auch die Betriebsgrößenstrukturen zum Verständnis dieser Schwerpunktbildung eine wichtige Rolle.

Die beigefügten Karten 1 und 2 zeigen auf Kreisbasis, daß die Förderung besonders die fränkischen und ostbayerischen Mittelgebirge, die Alpen und das Alpenvorland erreicht.

Ein hier nicht dargestellter Vergleich der in den einzelnen Landkreisen 1988/89/91 verteilten Prämiensummen belegt, daß sich die Schwerpunkte der Förderung kaum verändert haben. Anders allerdings 1996, wo die Aufgabe der begrenzenden Gebietskulisse wesentliche Fördermittel auch in nichtbenachteiligte Gebiete fließen ließ.

Bezüglich der durchschnittlich pro Betrieb gewährten Prämie nach Teil A stehen neben den nordostoberfränkischen Kreisen Hof und Wunsiedel vor allem die Landkreise der mittleren und westlichen bayerischen Alpen, die südlichen Landkreise um 
München und zahlreiche Stadtkreise weit über dem Durchschnitt. Betriebsgrößen und natürliches Ertragsprotential bieten erste regional differenzierte Erklärungsansätze. Bewirtschaftungsentgelt und Kulturlandschaftsprogramm A-Mittel werden im Durchschnitt mit 883,-DM bzw. 2788,-DM pro beteiligtem Betrieb für das Jahr 1996 anzusetzen sein. Generell gilt, daß mit steigender Betriebsgröße auch die Fördermittel beträchtlich zunehmen (vergl. Bayerischer Agrarbericht S. 84) "Die Abhängigkeit der Einkommenslage von den Direktzahlungen tritt deutlich zutage", eine Entwicklung, die in der gegenwärtigen wirtschaftlichen Situation oft nicht unkritisch beurteilt wird. Es soll auch nicht verschwiegen werden, daß die Höhe der Dirktzahlungen den Pachtflächenmakrt sehr nachteilig beeinflussen kann.

\section{b) Das Bayerische Vertragsnaturschutzprogramm}

Im Unterschied zur Zielsetzung der Agrarpolitik steht hier der Schutz der Natur und die Landschaftspflege im Vordergrund. Seit 1983 wurden unter dem Dach des Bayerischen Staatsministeriums für Landesentwicklung und Umweltfragen Naturschutzprogramme eingeführt, von denen 8 im Bayerischen Vertragsnaturschutzprogramm 1995 zusammengefaßt wurden. Unter Vertragsnaturschutz werden dort Vereinbarungen zwischen der unteren Naturschutzbehörde und Landwirten bzw. Eigentümern oder Nutzungsberechtigten landwirtschaftlich nutzbarer Flächen verstanden. Unter naturschutzfachlicher Anleitung gelten als Ziele (Richtlinien ...10)

- die nachhaltige Leistungsfähigkeit des Naturhaushaltes zu sichern und zu verbes sern - die Vielfalt, Eigenart und Schönheit von Natur und Landschaft als Lebens grundlage des Menschen zu sichern...

- die Lebensräume und Lebensgemeinschaften der heimischen Tier- und Pflanzenwelt zu erhalten...

Die Vereinbarungen betreffen "naturschonende Bewirtschaftungsweisen und Pflegemaßnahmen" sowie langfristige Flächenbereitstellung für Naturschutz und Landschaftspflege. Infolge der Laufzeit der Verträge (5 Jahre) konnten auch EU-Mittel eingebracht werden.

Das Programm sieht ein Entgelt für biotopspezifische Maßnahmen (Ackerflächen, Wiesen, Weiden, Streuobstbestände, Teiche, Weinberge, bzw. spezielle Artenschutzmaßnahmen) sowie für nichtbiotopspezifische Maßnahmen (Mager- und Trockenstandorte, Naturschutzgebiete usw.) vor (vgl. Tab. 2). Mit dem schon länger praktizierten Erschwernisausgleich für Feuchtflächen und dem Landschaftspflegeprogramm stehen aus diesem Ministerium für Naturschutzprogramme in Bayern 41Mill. DM zur Verfügung. Über 50.000 Hektar ökologisch bedeutsame Flächen waren vertraglich an dieses Programm 1995 gebunden (vgl. Tab. 3) mit regionalen Schwerpunkten in Oberbayern und Unterfranken. Wenn auch die in diesem Programm eingesetzten Mittel weit hinter den finanziellen Möglichkeiten des Landwirtschaftsministeriums zurückbleiben, so ist doch bei anderer Zielstellung auch hier die Mitarbeit der Landwirte an der Landschaftspflege gegeben. Eine weitere Aktivität belegt den Dienstleistungscharakter des primären Sektors. 


\section{c) Hinweise zur Landschaftspflege der Kreise/Gemeinden}

Dieser kurze Einblick in die durch eine geänderte Bewertung der Landschaft eingetretene Problemlage und die Reaktion der öffentlichen Hand muß dringend noch ergänzt werden durch einen wenigstens kurzen Hinweis auf das Engagement der Landkreise und der Gemeinden. Seit 1986 wurden z.B. sogenannte "Landschaftspflegeverbände" häufig auf Kreisebene gegründet, die die konkrete Durchführung von Pflegemaßnahmen organisieren. Unter kommunaler Beteiligung entscheiden politische Mandatsträger, Naturschützer und Landwirte über die Planung und Ausführung der praktischen Arbeiten, die von den Mitgliedern mit staatlicher Unterstützung finanziert werden.

Auf Gemeindebasis existieren mannigfache Initiativen zur Landschaftspflege, besonders in den Fremdenverkehrsgemeinden der Alpenländer. Unterschiedlich sind die Höhe des Leistungsentgeltes und die jeweiligen Bemessungsgrundlagen. Unterschiedliche Flächenbewirtschaftungsprämien beziehen sich zum Teil auf den Viehbestand, unterschiedliche Nutzung, Lage, Bearbeitbarkeit der zu pflegenden Grundstücke usw. Für die Mittelbereitstellung werden öfter Fremdenverkehrsbeiträge bzw. Kurabgaben herangezogen, aber auch Freiwassermengen für Landwirte usw. gewährt. Ein auch nur halbwegs vollständiger Überblick kann derzeit nicht gegeben werden, vgl. z.B. die Zusammenstellung für Österreich (11) oder bei W. Zeller (12). Auch die Arbeit der Maschinenringe muß in diesem Zusammenhang erwähnt werden, die neben den traditionellen Arbeiten innerhalb der Landwirtschaft immer mehr im Dienstleistungsbereich tätig sind. Die Landwirte

bringen hier neben ihrer Arbeitskraft und Maschinenausstattung eine detaillierte Kenntnis der zu pflegenden Flächen mit und können für die Gemeinden preisgünstig arbeiten.

Heute kann eine außerordentlich große Vielfalt von Pflegemaßnahmen durch die Öffentliche Hand, aber auch auf privatwirtschaftlicher Basis beobachtet werden. Dies zeigt, daß die Landschaftspflege innerhalb der letzten Jahrzehnte immer mehr als Notwendigkeit empfunden und anerkannt wurde. Die Lösung dieser Problematik setzt voraus, daß im Selbstverständnis der Landwirtschaft Pflegeleistungen und Sicherung der natürlichen Lebensgrundlagen den gleichen Stellenwert einnehmen wie die Nahrungsmittelproduktion. 
Bayerisches Kulturlandschaftsprogramm 1996

Karte 1: Teil A - Prämien pro Betrieb

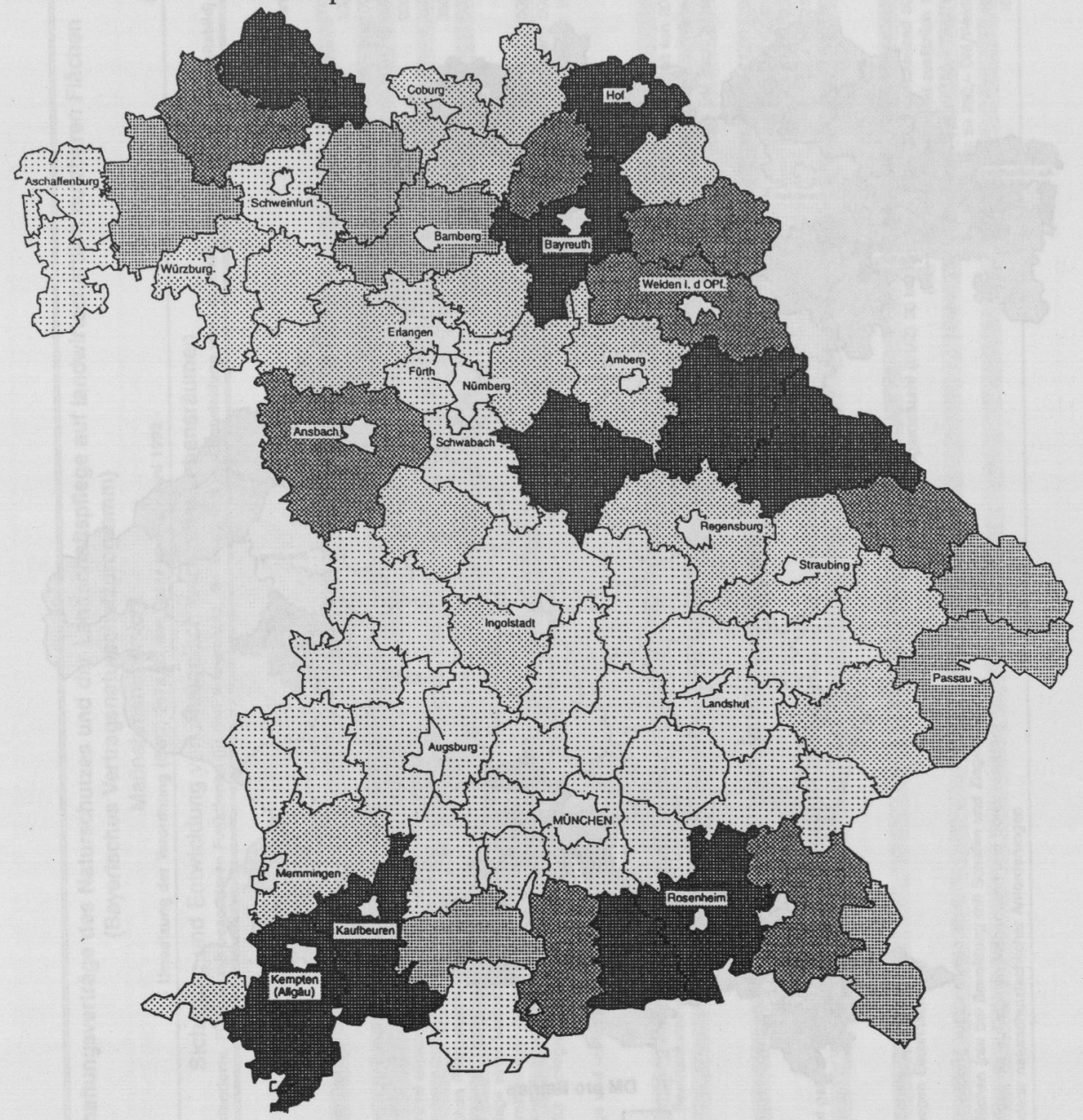

Mio. DM pro Landkreis

0,3 bis u. 1,0
1,0 bis u. 1,8
1,8 bis u. 2,3
2,3 bis u. 3,0
3,0 u. mehr
m.

\footnotetext{
Quelle: Bayer. Staatsmin, fûr

Emährung, Landwirtschaft

und Forsten

Entwurt: K. Ruppert

Kartographie: H. Sladkowski

München, 1997
} 
Bayerisches Kulturlandschaftsprogramm 1996

Karte 2: Teil B -

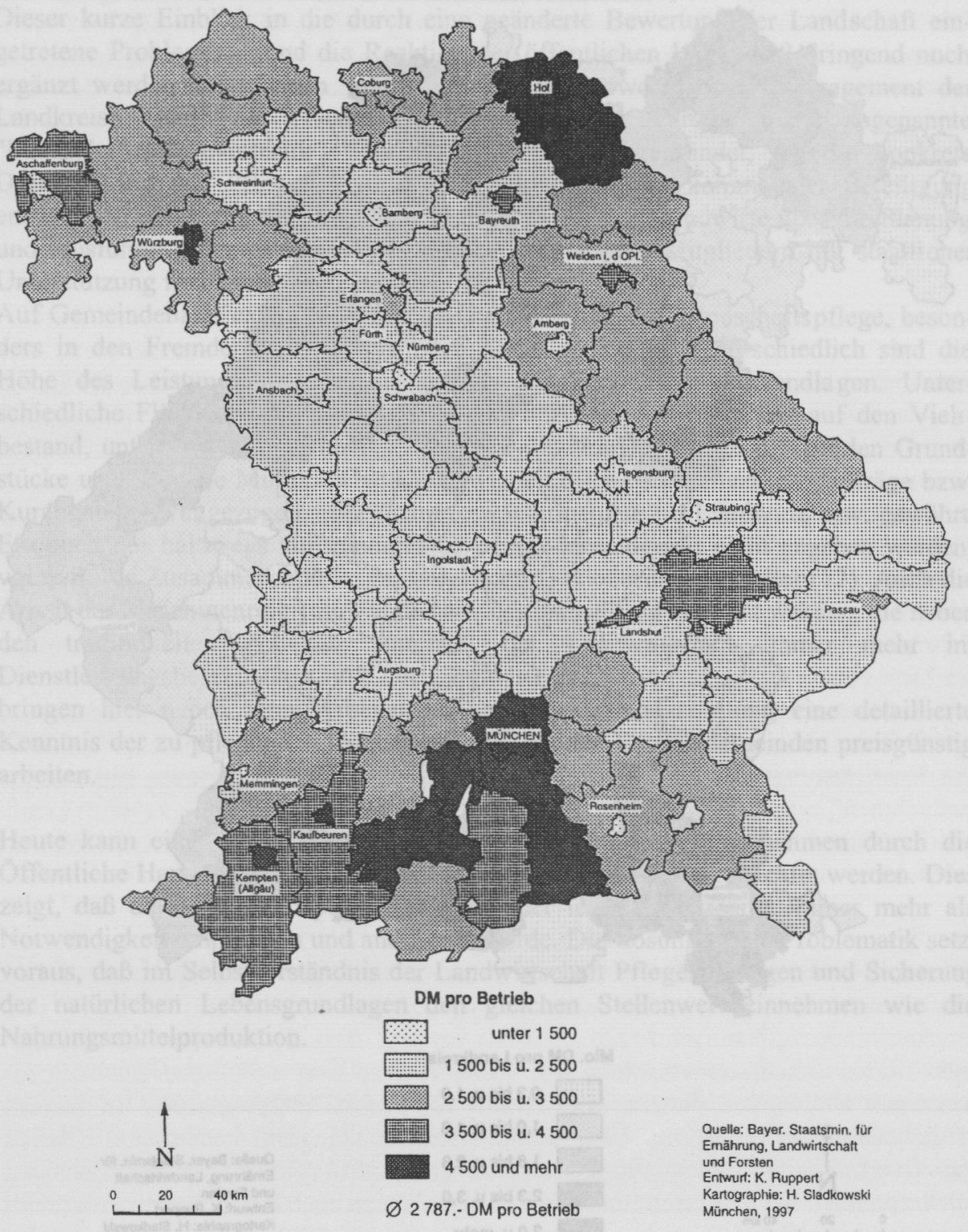




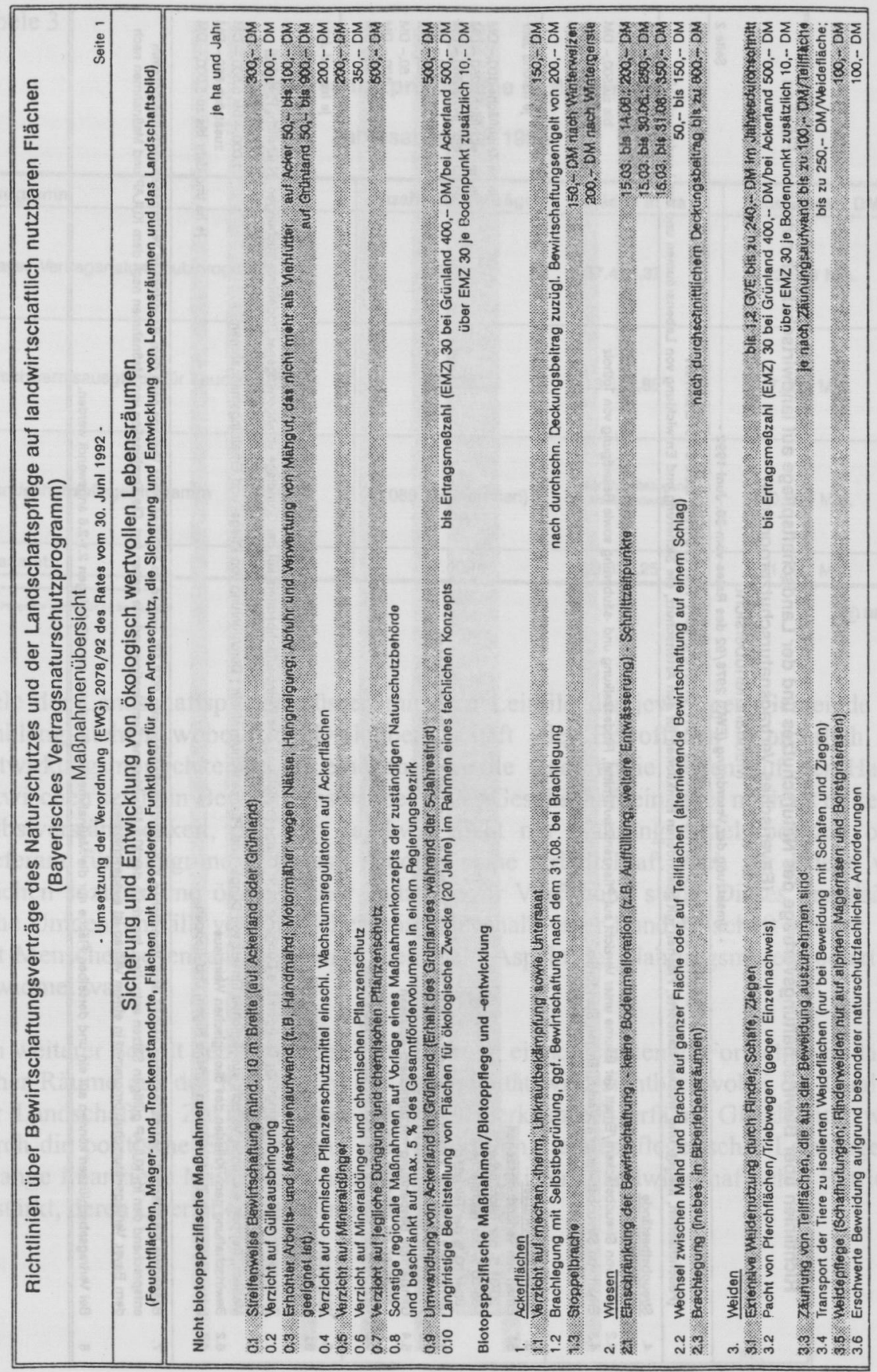




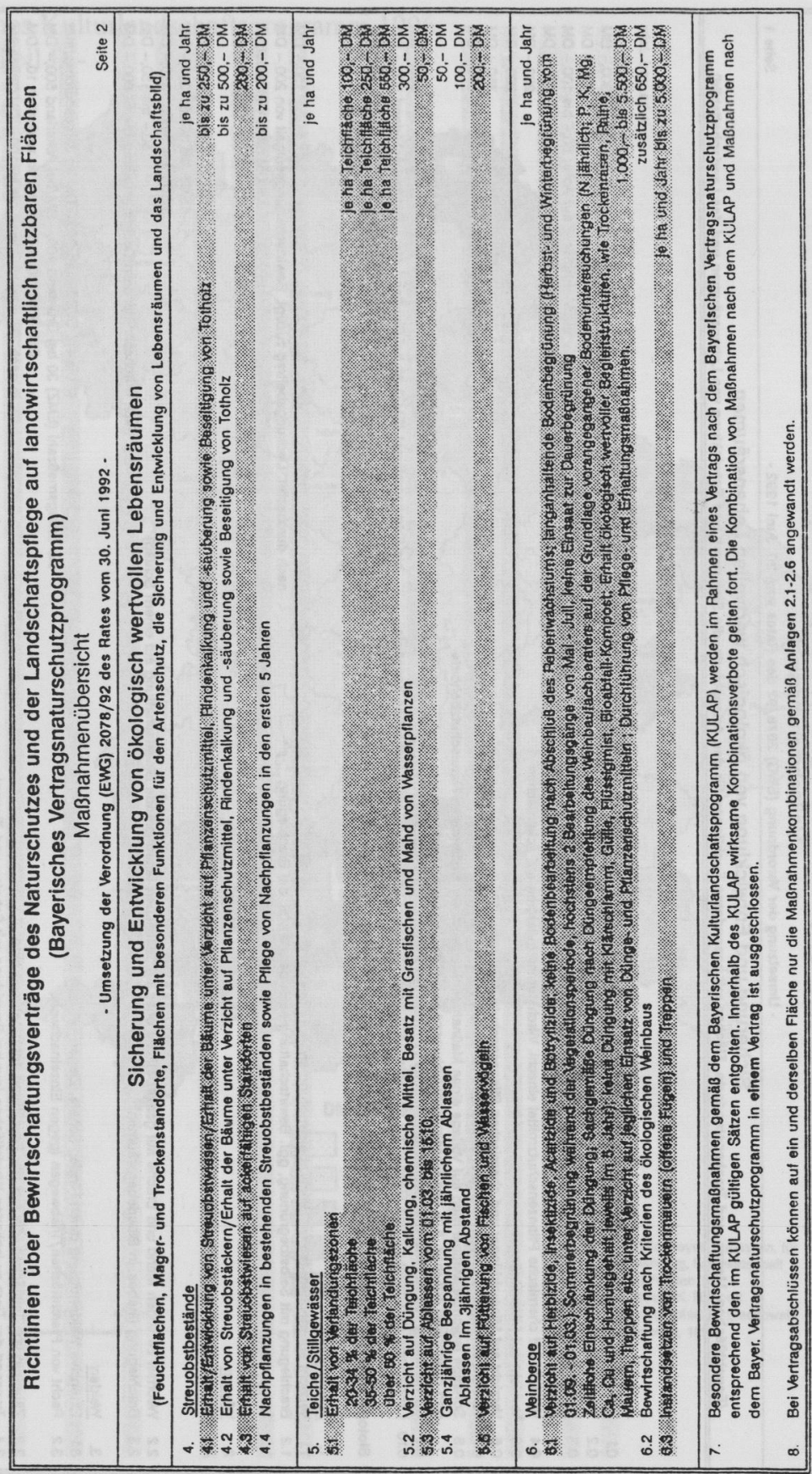


Tabele 3

\section{Naturschutzprogramme in Bayern}

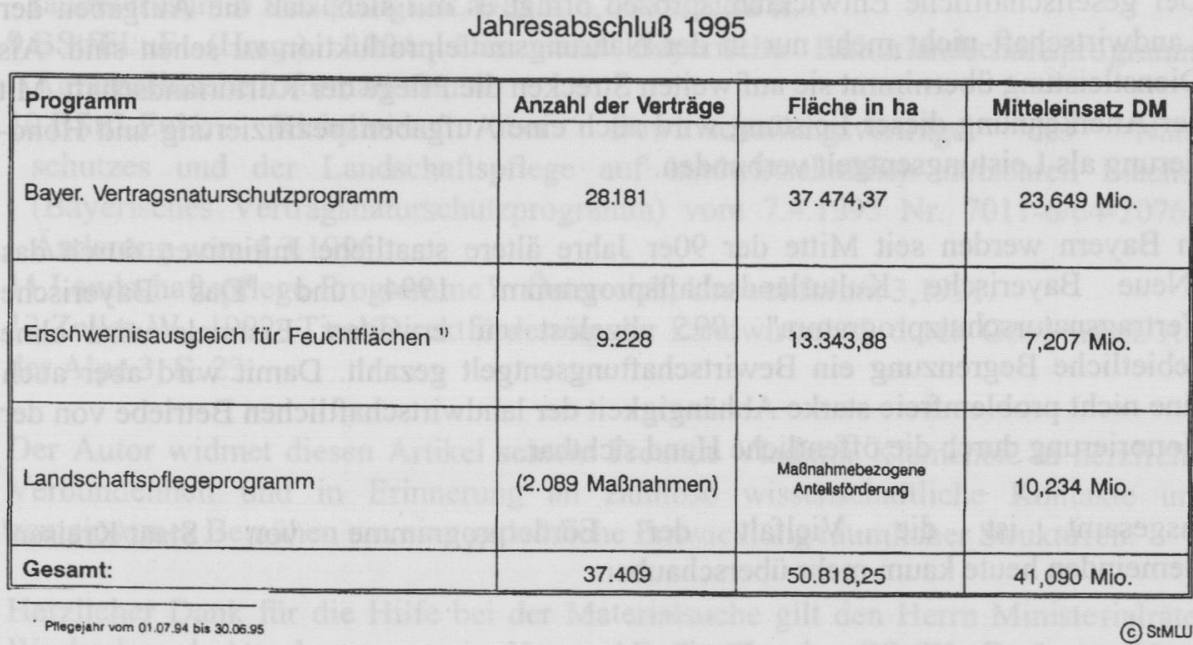

Ziele der Landschaftspflege müssen mit dem Leitbild der jeweiligen Gemeinde im Einklang stehen, wobei eine Dialogbereitschaft aller Betroffenen erforderlich ist. Mitwirkungsmöglichkeiten für eine angewandte Geographie liegen auf der Hand. Inzwischen setzt ein Bewußtseinswandel in der Gesellschaft ein, aber noch ist es keine Selbstverständlichkeit, daß der Landwirt nicht nur Nahrungsmittelproduzent oder Lieferant für Baugrundstücke ist, sondern seine Arbeitskraft auch zur Pflege von Flächen sozialer und ökologischer Nutzung zur Verfügung stellt. Dieses grundsätzliche Umdenken fällt verständlicherweise innerhalb einer Landwirtschaft schwer, die seit Menschengedenken einzig und allein dem Aspekt der Nahrungsmittelproduktion gewidmet war.

Ein weiterer Schritt der regionalen Entwicklung, ein konsequentes Fortschreiten ländlicher Räume aus der Ruralität in die Regionalität wird sichtbar, wobei die Qualität der Landschaft in Zukunft immer mehr Aufmerksamkeit erfährt. Gleichzeitig wird durch die politische Entscheidung der Honorierung landespflegerischer Leistungen solange finanzielle Mittel vorhanden sind - die Existenz landwirtschaftlicher Betriebe gestärkt, deren Überleben sonst infrage gestellt wäre. 


\section{ZUSAMMENFASSUNG}

Der gesellschaftliche Entwicklungsprozeß bringt es mit sich, daß die Aufgaben der Landwirtschaft nicht mehr nur in der Nahrungsmittelproduktion zu sehen sind. Als Dienstleistung übernimmt sie auf weiten Strecken die Pflege der Kulturlandschaft. Mit der Anerkennung dieser Leistung wird auch eine Aufgabenspezifizierung und Honorierung als Leistungsentgelt verbunden.

In Bayern werden seit Mitte der 90er Jahre ältere staatliche Initiativen durch das "Neue Bayerische Kulturlandschaftsprogramm" 1994 und "Das Bayerische Vertragsnaturschutzprogramm" 1995 abgelöst und erweitert. Erstmals wird ohne gebietliche Begrenzung ein Bewirtschaftungsentgelt gezahlt. Damit wird aber auch eine nicht problemfreie starke Abhängigkeit der landwirtschaftlichen Betriebe von der Honorierung durch die öffentliche Hand sichtbar.

Insgesamt ist die Vielfalt der Förderprogramme von Staat/Kreisen/ Gemeinden heute kaum mehr überschaubar.

\section{LITERATURHINWEISE}

1.Klemenčič V., 1986: Sozialgeographische Probleme der Arbeiter-Bauern-Struktu ren unter besonderer Berücksichtigung der Situation in Jugoslawien. Münchner Studien zur Sozial- und Wirtschaftsgeographie Bd. 4, S. 75 ff.

2.Haber W., 1978: Grundsätzliche Anmerkungen zum Problem der Pflege der Landschaft. Tagungsber. Akad. für Naturschutz 5, S. $87 \mathrm{ff}$.

Priewasser R.,: Agrarische Landschaftspflege Wirkungen auf die Umweltqualität von Erholungsräumen und Abgeltungsfragen. DISP 105, S. $15 \mathrm{ff}$.

Gießübel R., 1988: Ausgleichszahlungen an die Landwirtschaft. ARL, Hannover.

Mayerl D., 1990: Die Landschaftspflege im Spannungsfeld zwischen gezieltem Eingreifen und natürlicher Entwicklung. Natur u. Landschaft 4, S. 167 ff.

Landschaftspflege als Aufgabe der Landwirte und Landschaftsgärtner, Laufener Seminarbeiträge $1 / 1980$.

3.Ruppert K., 1985: Freizeitverhalten als Flächennutzung. Klagenfurter Geographische Schriften 6, S. 93-106.

4.Ammer U., Pröbstl U., 1991: Freizeit und Natur. Hamburg.

5.Pevetz W., 1989: Landwirtschaft in Naturschutz und Landschaftspflege. Bundesanstalt für Agrarwirtschaft, Schriftenreihe Nr. 56, Wien.

6.Ruppert K., Deuringer L. Maier J., 1971: Das Bergbauerngebiet der deutschen Alpen WGI-Berichte z. Regionalforschung 7. 
7.BStfELuF (Hrsg.), 1996: Bayerischer Agrarbericht 1996. München.

BMfELuF (Hrsg.), 1997: Agrarbericht der Bundesregierung 1997, Bonn.

8.Zum Stand Ende der 80er Jahre vgl. K. Ruppert: Agrarlandschaft unter dem Einfluß raumwirksamer Staatstätigkeit, DELA 6, 1989, S 80 ff.

9.BStfELuF (Hrsg.), 1994: Das neue Bayerische Kulturlandschaftsprogramm, Agrarpolitische Informationen 4.

10.BStfLEuU: Richtlinien über Bewirtschaftungsverträge des Natur schutzes und der Landschaftspflege auf landwirtschaftlich nutzbaren Flächen (Bayerisches Vertragsnaturschutzprogramm) vom 7.4.1995 Nr. 7011-6/64-20766, Änderung vom 4.3.1996.

11 Landschaftspflege-Programme in Österreich, Umweltforum 3,1991.

12.Zeller W.,,1992: Tirol:Direktförderung der Landwirtschaft durch Gemeinden. Auf der Alpe 3, S. 23.

Der Autor widmet diesen Artikel seinem Freunde Vladimir Klemenčič in herzlicher Verbundenheit und in Erinnerung an zahllose wissenschaftliche Kontakte und gemeinsames Bemühen um eine gedeihliche Entwicklung räumlicher Strukturen.

Herzlicher Dank für die Hilfe bei der Materialsuche gilt den Herrn Ministerialräten Wackerl und Attenberger sowie Herrn AR Treftler im BStfELuF, ferner Herrn Min.Rat Eisenried, BStfEuU in München.

\section{UREJANJE KRAJINE V LUČI APLIKATIVNE GEOGRAFIJE}

\section{Povzetek $^{1}$}

Že pred 30 leti je jubilant v zborniku posvečenem Hartke-ju zapisal, da je "prostor ogledalo družbenega razvoja". Zapisano ugotovitev je utemeljeval na primerih preslajanja klasične v industrijsko družbo na Slovenskem. Rast tamkajšnjih delavskokmečkih gospodinjstev je posledično spremenila rabo in funkcijo površin $\mathrm{v}$ agrarni rabi in vplivala na preoblikovanja podeželja nasploh. Na Slovenskem, kot tudi drugod v Evropi, je obsežna socialno-geografska preslojitev posledično udejanila naslednje:

- funkcija prostega časa je pridobila enakovreden tretma v sklopu preostalih: dela, (pre)bivanja, pre/oskrbovanja, ...;

- nove oblike prostorske (iz)rabe so, skladno z dvojnostjo bivanja (počitniška bivališča), odločilno (pre)oblikovale podeželje;

\footnotetext{
${ }^{1}$ Pripravil in prevedel dr. Anton Gosar
} 
- porast zavedanja okoljske problematike in naravovarstvene zahteve so podeželjskemu in mestnemu prostoru dale povsem novo funkcijo in podobo.

Podeželjska pokrajina je vedno pogosteje videna kot rekreacijski potencial, kot prostor $v$ katerem je moč udejanjati različne aktivnosti $v$ (dela) prostem času. Vse oblike rabe prostega časa smemo, tako kot kmetijstvo (svojčas in deloma tudi danes) uvrščati $v$ dejavnost (iz)rabe površin na podeželju. Intenzivnost te rabe je posledica različnih dejavnikov naravnogeografskega in socialnogeografskega značaja. Raziskave na obrobju Münchna so denimo pokazale, da obiskovalci pogosteje obiskujejo gozdne jase in gozdni rob kot obsežnejše, strnjene z gozdom zarasle površine. Nekateri raziskovalci menijo, da je 50-70\% delež gozda razmerje, ki odgovarja izletnikom. Zavedati se moramo, da naraščajoča rekreativna dejavnost (enakovrednost drugim funkcijam življenja!) lahko ogrozi ravnovesje $\mathrm{v}$ prostoru $\mathrm{v}$ katerem se udejanjajo socialno-geografske funkcije.

Podobno lahko preslojitveni procesi vplivajo na izgled in rabo pokrajine. Ekvilibrij med kmetijsko in prostočasnimi dejavnostmi obeta zaustavitev procesa degradacije pokrajine iz strani obeh dejavnikov preobrazbe podeželja. Kmetu je potrebno priznati njegov delež pri urejanju in negovanju kulturne pokrajine. Taka pokrajina sme, posledično, v vsej svoji pestrosti služiti (tudi) skrbno zasnovanim prostočasnim dejavnostim. Okoljevarstveni principi morajo biti sestavni del tako zasnovanega modela. Že na začetku osemdesetih let so na Bavarskem za negovanje krajine namenili letno okrog $3-5 \%$ deželnih investicij, te pa so zadevale okrog $10 \%$ kmetijskih površin. Sredi devetdesetih let se v Nemčiji s tovrstnim investicijskim denarjem neguje, oplaja oziroma izboljšuje kar $20 \%$ do $50 \%$ podeželjske pokrajine.

V nadaljevanju članka avtor podrobno predstavi organizacijske oblike pokrajinskega negovanja na Bavarskem. Predvsem izpostavlja pozitivne učinke te politično vodene akcije, ki utrjuje podeželje v socialnogeografskem in prostočasnem pogledu. Kmetu je zagotovljena eksistenca navkljub, iz strani (evropske) federacije diktirani, limitirani kmetijski proizvodnji. Na Bavarskem izvajajo dvoje v negovanje kulturne krajine usmerjenih zakonov. Ne da bi upoštevala pokrajinske dejavnike/omejitve (pri kmetijski produkciji) namenja dežela kmetom sredstva za negovanje lastnih, kmetijskih površin. Sredstva dežele, države in federacije že zdavnaj presegajo prihodke, ki jih kmetje ustvarjajo s proizvodnjo hrane. To (nam) daje nova izhodišča za razpravo.

Današnji podeželjski prostor moramo videti in obravnavati kompleksno, v vsej njegovi funkcijski in prostorski pestrosti. Predvsem ne sme postati predmet izključno proizvodne funkcije/obravnave. $\mathrm{V}$ času postindustrijske družbe je $\mathrm{v}$ tem prostoru opaziti enakovredno prisotnost storitvenih dejavnostmi - turizma/rekreacije. Ta je (dohodkovno) lahko zanimiva toliko časa dokler ohranja kulturno (po)krajino 
negovano. Negovanju odmerja država/družba ustrezna sredstva, katerih pretiran dotok sproža nove dileme in probleme. 